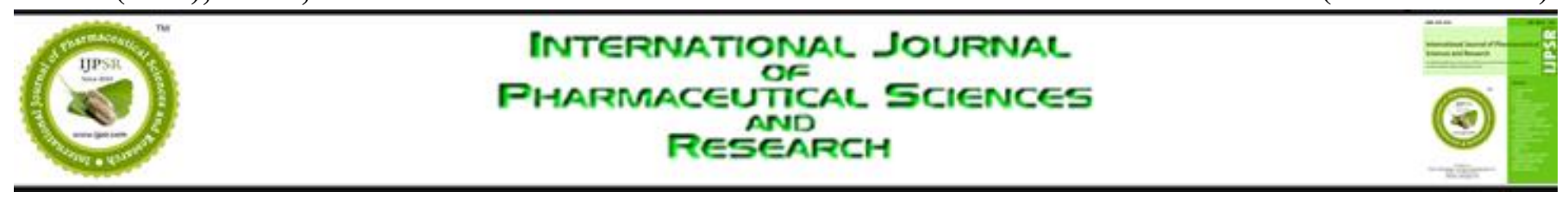

Received on 11 January, 2014; received in revised form, 23 April, 2014; accepted, 26 May, 2014; published 01 July, 2014

\title{
FLOATING DRUG DELIVERY SYSTEM: A REVIEW
}

\author{
Vandana Tiwari* $^{1}$, Anurag Verma ${ }^{1}$ and Navneet Verma ${ }^{2}$
}

Department of Pharmaceutics, Institute of Pharmaceutical Sciences and Research (IPSR), ${ }^{1}$ Sohramau, Unnao, Uttar Pradesh, India

Department of Pharmacy, Institute of Foreign Trade and Management (IFTM) ${ }^{2}$, Moradabad- 244001, Uttar Pradesh, India

Keywords:

Gastric residence time, Gastroretentive drug delivery system (GRDDS), Floating drug delivery system (FDDS), In-vitro evaluation of floating drug delivery system

Correspondence to Author:

Vandana Tiwari

Assistant Professor, Department of Pharmaceutics, Institute of

Pharmaceutical Sciences and

Research (IPSR), Sohramau, Unnao, Uttar Pradesh, India

E-mail: vandystudy@gmail.com

\begin{abstract}
In the recent years, scientific and technological advancements have been made in the research and development of novel drug delivery systems by overcoming physiological troubles such as short gastric residence times and unpredictable gastric emptying times. Gastric emptying of dosage form is extremely variable process and ability to prolong and control the emptying time. Gastric transit time is valuable asset for dosage forms, which reside in the stomach for a long period of time than conventional dosage form. Several approaches are currently utilized in the prolongation of the gastric residence times, including floating drug delivery systems, swelling and expanding systems, polymeric bioadhesive systems, modified-shape systems, high-density systems and other delayed gastric emptying devices. The purpose of writing this review on floating drug delivery systems (FDDS) was to compile the recent literature with special focus on the principal mechanism of floatation to achieve gastric retention. This review explains briefly about types of floating system, advantages, limitation, floating mechanism, factors affecting floating system, drug candidates suitable for floating, evaluation parameters and application of the system.
\end{abstract}

INTRODUCTION:
advancement in drug delivery, oral route of administration has received the more attention and success because the gastrointestinal physiology offers more flexibility in dosage form design than other routes. Hence, research continuously keeps on searching for ways to deliver drugs over an extended period of time, with a well-controlled release profile.

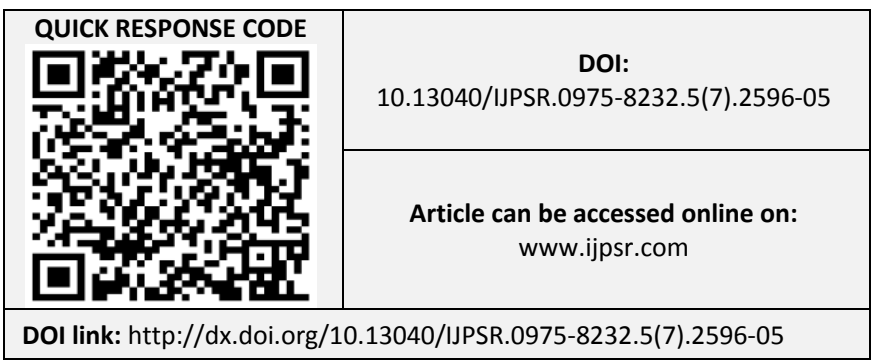

Gastric emptying of dosage form is extremely variable process and ability to prolong and control the emptying time. Gastric transit time is valuable asset for dosage forms, which reside in the stomach for a long period of time than conventional dosage form. Conventional oral dosage forms such as tablets, capsules provide specific drug concentration in systemic circulation without offering any control over drug delivery and also cause great fluctuations in plasma drug levels. Many attempts have been made to develop sustained release preparations with extended clinical effects and reduced dosing frequency. A problem frequently encountered with conventional sustained release dosage forms is the inability to increase their residence time in stomach and no control over drug delivery, leading to fluctuations 
in plasma drug level. ${ }^{1}$ Gastric emptying occurs during fasting as well as fed states. The pattern of motility is however distinct in the 2 states. During fasting state inter digestive series of electrical events take place which cycle both through stomach and intestine every 2 to 3 hours. This is called the inter digestive myloelectric cycle or migrating myloelectric cycle (MMC), which is further divided into following 4 phases (fig. 1) as described by Wilson and Washington.

i. Phase I (basal phase) lasts from 40 to 60 minutes with rare contractions.

ii. Phase II (pre-burst phase) lasts for 40 to 60 minutes with intermittent action potential and contractions. As the phase progresses the intensity and frequency also increases gradually.

iii. Phase III (burst phase) lasts for 4 to 6 minutes. It includes intense and regular contractions for short period. It is due to this wave that all the undigested material is swept out of the stomach down to the small intestine. It is also known as the housekeeper wave.

iv. Phase IV lasts for 0 to 5 minutes and occurs between phases III and I of 2 consecutive cycles $^{2}$.

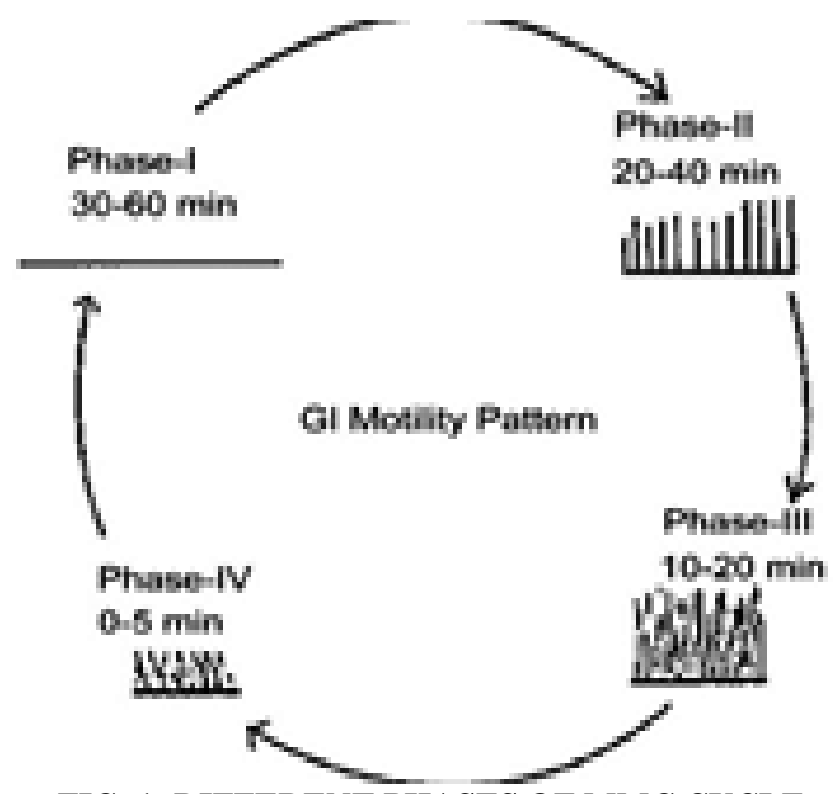

FIG. 1: DIFFERENT PHASES OF MMC CYCLE
After the ingestion of a mixed meal, the pattern of contractions changes from fasted to that of fed state. This is also known as digestive motility pattern and comprises continuous contractions as in phase II of fasted state.

These contractions result in reducing the size of food particles (to less than $1 \mathrm{~mm}$ ), which are propelled toward the pylorus in a suspension form. During the fed state onset of MMC is delayed resulting in slowdown of gastric emptying rate ${ }^{3}$.

Gastroretentive systems can remain in the gastric region for several hours and hence significantly prolong the gastric residence time of drugs. Prolonged gastric retention improves bioavailability, reduces drug waste and improves solubility for drugs that are less soluble in a high $\mathrm{pH}$ environment.

Gastric retention may increase solubility for the drugs which are poorly soluble in intestine due to alkaline $\mathrm{pH}$ before they are emptied, resulting in improved bioavailability. These systems are also advantages in improving GIT absorption of a drug with narrow absorption windows as well as for controlling release of those drugs which are having site specific absorption limitations.

From the formulation and technological point of view, floating drug delivery system (FDDS) is considerably easy and logical approach in of development gastroretentive drug delivery system (GRDDS).

Approaches to GRDDS: Over the last three decades, various approaches have been pursued to increase the retention of an oral dosage form in the stomach, including (fig. 2);

- Floating system,

- Swelling and expanding systems,

- Bioadhesive systems,

- Modified-shape systems,

- High-density systems and;

- Other delayed gastric emptying devices. 


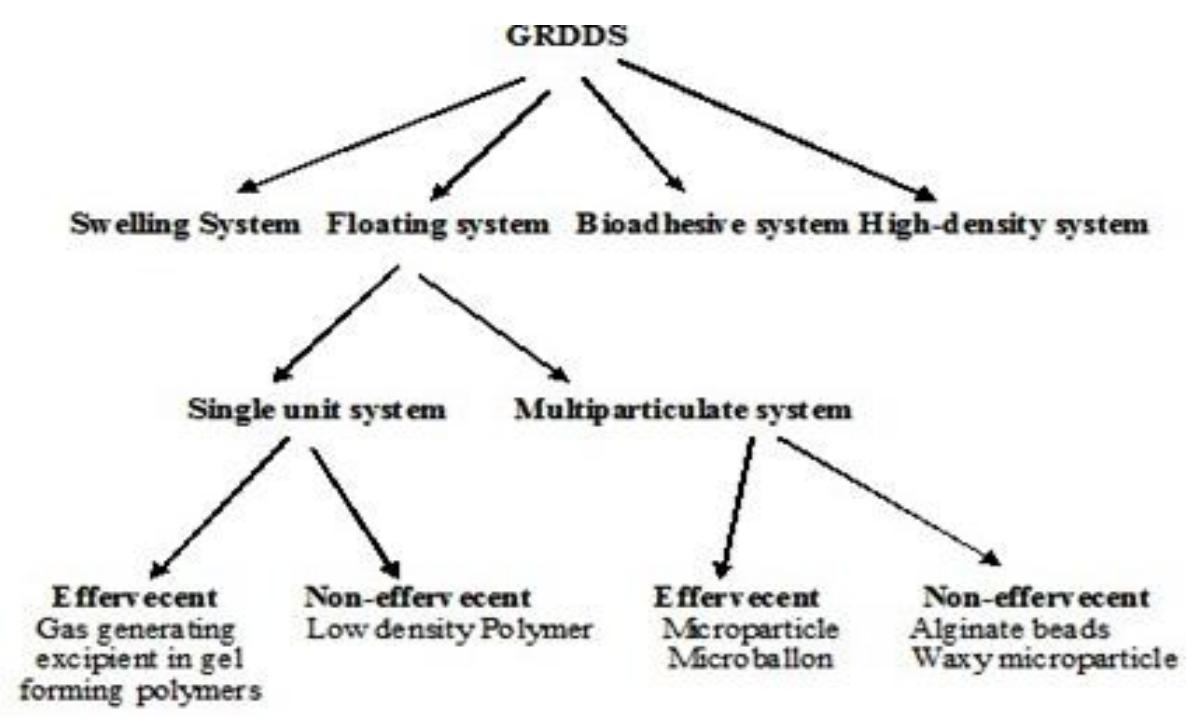

FIG. 2: FLOWCHART ENLISTING VARIOUS APPROACHES USED FOR DESIGNING FDDS

a) Hydrodynamically Balanced Systems (HBS): HBS have a bulk density lower than gastric fluids and thus remain buoyant in the stomach without affecting the gastric emptying rate for a prolonged period of time. While the system is floating on the gastric contents, the drug is released slowly at a desired rate from the system. After the release of drug, the residual system is emptied from the stomach. This results in an increase in the GRT and a better control of fluctuations in plasma drug concentrations in some cases. The incorporated buoyant materials enable the device to float (fig. 3).

\section{HBS $^{\text {TM }}$ - MODE OF ACTION}

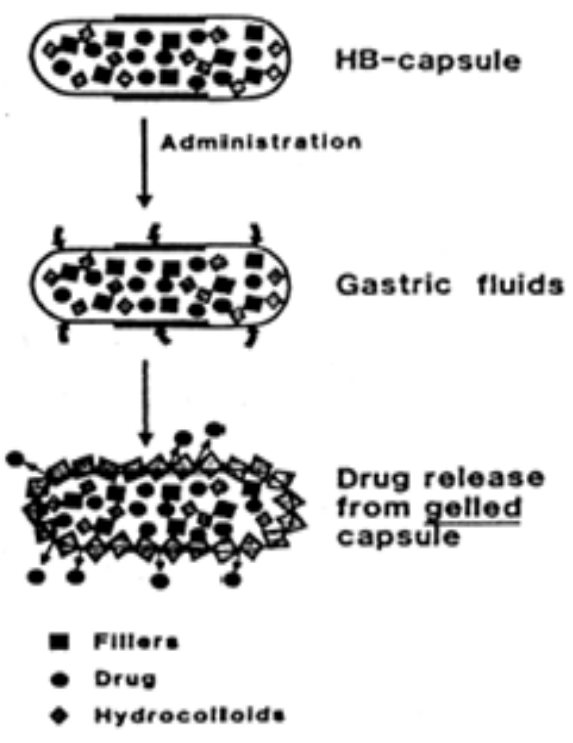

FIG. 3: HYDRO-DYNAMICALLY BALANCED SYSTEM (CAPSULE SYSTEM) b) Raft systems incorporating Alginate Gels: These have a carbonate component and, upon reaction with gastric acid, bubbles form in the gel, enabling floating.

c) Bioadhesive or Mucoadhesive systems: These systems are used to localize a delivery device within the lumen and cavity of the body to increase the drug absorption process in a site specific manner (fig. 4). In these approaches involve the use of bioadhesive polymer are used that can be adhere to the epithelial surface of GIT. The proposed mechanism of bioadhesive is the formation of hydrogen and electrostatic bonding at the mucus polymer boundary. The basis of adhesion in that a dosage form can stick to the mucosal surface by different mechanism. These mechanisms are:

1) The wetting theory, which is based on the ability of bioadhesive polymers to spread and develop intimate contact with the mucous layers.

2) The diffusion theory which proposes physical entanglement of mucin strands the flexible polymer chains, or an interpenetration of mucin strands into the porous structure of the polymer substrate.

3) The absorption theory, suggests that bioadhesion is due to secondary forces such as Vander Waal forces and hydrogen bonding. 
4) The electron theory, which proposes attractive electrostatic forces between the glycoprotein mucin network and the bio adhesive material.

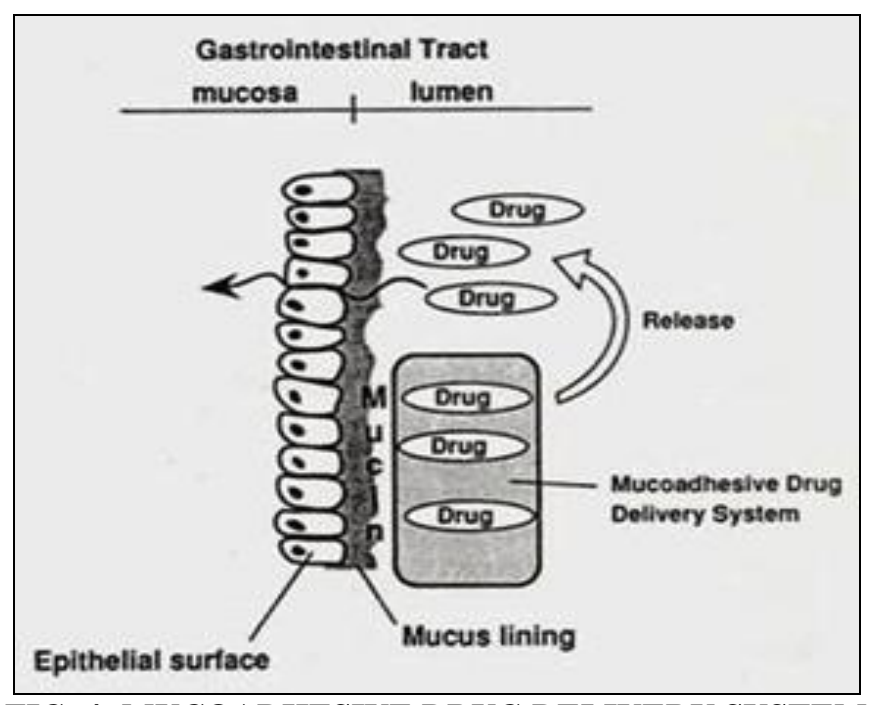

FIG. 4: MUCOADHESIVE DRUG DELIVERY SYSTEM

d) Modified shape system: These are nondisintegrating geometric shapes molded from silastic elastomer from polyethylene blends and extended the gastric transit time (GTT) depending on the size, shape and flexural modulus of drug delivery device. Rapid hydration in contact with the mucoepithelial surface appears to favor adhesion, particularly if water can be excluded at the reactive surfaces.

e) High Density Systems: They include coated pallets and have density greater than that of the stomach content $(1.004 \mathrm{gm} / \mathrm{cm})$. This goal is achieved by coating the drug with a heavy inert material such as barium sulphate, $\mathrm{ZnO}$, titanium dioxide. This formulation of high-density pellet is based on assumption that heavy pellets might remain longer in the stomach, since they are position in the lower part of the antrum.

f) Swelling System: These types of products swell to an extent that prevents their exit from the stomach through the pylorus. Swelling delivery systems are capable of swelling to a size that prevents their passage through the pylorus. Upon coming in contact with gastric fluid, the polymer imbibes water and swells; as a result the dosage form is retained in the stomach for a longer period of time. This dosage form retained in the stomach for a longer these as a "Plug type system", since they exhibit tendency to remain logged in the pyloric sphincters.

g) Magnetic Systems: These are the systems which include external stimuli as magnetic field for site specific drug delivery. Some magnetically active compounds are incorporated in the dosage form to achieve site specificity.

h) Floating Drug Delivery Systems: Floating systems are low density systems that have sufficient buoyancy to float over the gastric contents and remain in the stomach for a prolonged period. While the system floats over the gastric contents, the drug is released slowly at the desired rate, which results in increased gastro-retention time and reduces fluctuation.

FDDS can be divided into non-effervescent and gas-generating (effervescent) system-

Non-effervescent Systems: This type of system, after swallowing, swells via imbibition of gastric fluid to an extent that it prevents their exit from the stomach. The formulation methods of such type dosage forms involves the mixing of the drug with a gel, which swells when comes in contact with gastric fluid and maintains a relative integrity of shape and a bulk density of less than one within the outer gelatinous barrier. The air trapped by the swollen polymer provides buoyancy these dosage forms. The most commonly used excipients in these systems include hydroxypropyl methyl cellulose (HPMC), poly acrylate polymers, polyvinyl acetate, carbopol agar, sodium alginate, calcium chloride, polyethylene oxide and polycarbonates. This system can be further divided into four sub-types:

1. Colloidal Gel Barrier System: These types of systems contain drug with gel-forming hydrocolloids which allow them to remain buoyant on the stomach content. This prolongs GRT and maximizes the amount of drug at its absorption sites in the solution form for ready absorption. This system incorporates a high level of one or more gel-forming highly soluble cellulose type hydrocolloid as hydroxypropyl 
cellulose, hydroxyethyl cellulose. This hydrocolloid hydrates and forms a colloid gel barrier around its surface after coming in contact with gastric fluid and also helps in sustain releasing of drug.

2. Microporous compartment system: In this technology a drug reservoir is encapsulated inside a micro porous compartment with pores along its top and bottom walls. The peripheral walls of the drug reservoir compartment are completely sealed. This sealing prevents any direct contact of gastric surface with the undissolved drug. The flotation chamber containing the delivery system to float over the gastric content entrapped air allows, in the stomach. Gastric fluid enters through an aperture, dissolves the drug and carries the dissolved drug for continuous transport across the intestine for absorption.

3. Alginate beads: - To develop Multi-unit floating dosage forms the freeze dried calcium alginate has been used. Spherical beads of approximately $2.5 \mathrm{~mm}$ in diameter can be prepared by the precipitation of calcium alginate via dropping sodium alginate solution into aqueous solution of calcium chloride. The beads are then separated, snap-frozen in liquid nitrogen, and freeze-dried at $-40^{\circ} \mathrm{C}$ for 24 hours, it leads to the formation of a porous system which can maintain a floating force for over 12 hours. These floating beads prolonged residence time for more than 5.5 hours (fig. 5).

4. Hollow microspheres / Microballons: A novel emulsion solvent diffusion method used to prepare hollow microspheres loaded with drug in their outer polymer shelf ethanol/ dichloromethane solution of the drug and an enteric acrylic polymer was poured into an agitated solution of poly vinyl alcohol (PVA) that was thermally controlled at $40^{\circ} \mathrm{C}$. The gas phase is generated in the dispersed polymer droplet by the evaporation of dichloromethane formed in the internal cavity of microsphere of the polymer and drug. The micro balloon floated continuously over the surface of an acidic dissolution media containing surfactant for more than $12 \mathrm{~h}$.

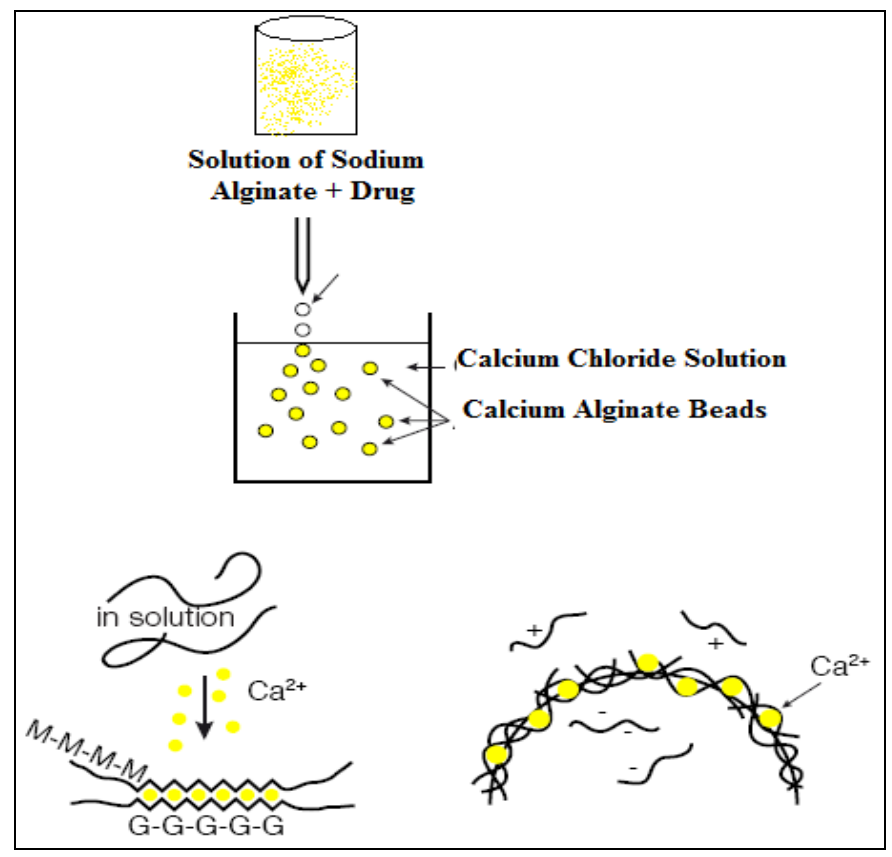

FIG. 5: MECHANISM OF GEL FORMATION THROUGH THE INTERACTION BETWEEN CALCIUM IONS AND SODIUM ALGINATE

Effervescent systems: These buoyant systems utilize matrices prepared with swell able polymers such as methocel polysaccharides (e.g., chitosan) and effervescent components (e.g., sodium bicarbonate, citric acid or tartaric acid. The system is so prepared that when it arrives in the stomach carbon dioxide is released, causing the formulation to float in the stomach.

Volatile liquid containing systems: The GRT of a drug delivery system can be sustained by incorporating an inflatable chamber which contains a liquid e.g. ether, cyclopentane, that gasifies at body temperature to cause the inflation of the chamber in the stomach. The device may also consist of a bioerodible plug made up of PVA, Polyethylene, etc. that gradually dissolves causing the inflatable chamber to release gas and collapse after a predetermined time to permit the spontaneous ejection of the inflatable systems from the stomach (fig. 6).

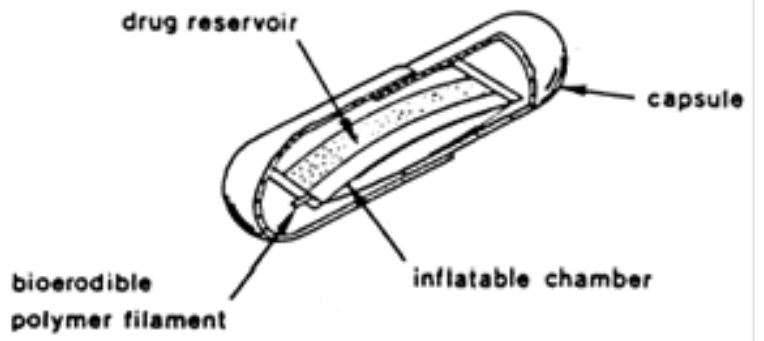

FIG. 6: VOLATILE LIQUID CONTAINING SYSTEM 
Gas-generating Systems: The effervescent reactions between carbonate/bicarbonate salts and citric/tartaric acid to liberate $\mathrm{CO}_{2}$ occurred in this delivery system, which gets entrapped in the gelled hydrocolloid layer of the systems thus decreasing its specific gravity and making it to float over chime. These systems contain matrices prepared with swellable polymers like methocel, polysaccharides like chitosan, effervescent components like sodium bicarbonate, citric acid and tartaric acid. The optimal stoichiometric ratio of citric acid and sodium bicarbonate for gas generation is reported to be $0.76: 1$.

The common approach used for the preparation of these systems involves resin beads loaded with bicarbonate and coated with ethyl cellulose. The coating, which is insoluble but permeable, allows permeation of water. Thus carbon dioxide is released, causing the beads to float in the stomach .Other reported approaches and materials that have been reported are highly swellable hydrocolloids and light mineral oils, a mixture of sodium alginate and sodium bicarbonate, multiple unit floating pills that generate carbon dioxide when ingested, floating mini-capsules with a core of sodium bicarbonate, lactose and polyvinyl pyrrolidone coated with hydroxypropyl methylcellulose (HPMC), and floating systems based on ion exchange resin technology etc. (fig. 7).
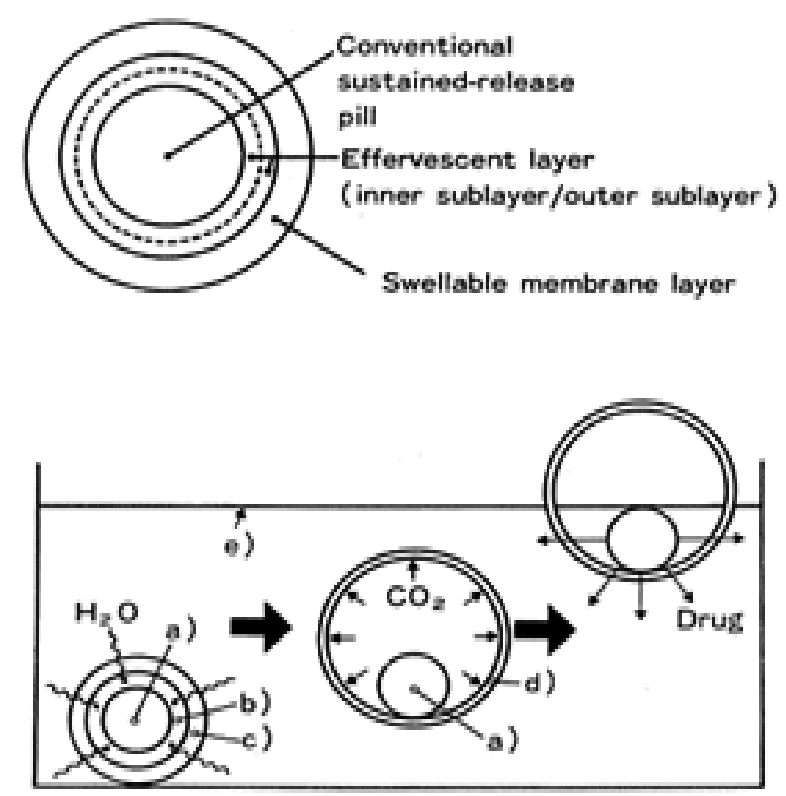

A

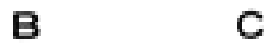

FIG. 7: EFFERVESCENT (GAS GENERATING) SYSTEM

\section{Advantages of Floating Drug Delivery:}

a) Enhanced bioavailability: The bioavailability of some drugs (e.g. riboflavin and levodopa) CR-GRDF is significantly enhanced in comparison to administration of non-GRDF CR polymeric formulations.

b) Enhanced first-pass biotransformation: When the drug is presented to the metabolic enzymes (cytochrome P-450, in particular CYP-3A4) in a sustained manner, the presystemic metabolism of the tested compound may be considerably increased rather than by a bolus input.

c) Sustained drug delivery/reduced frequency of dosing: The drugs having short biological half-life, a sustained and slow input from FDDS may result in a flipflop pharmacokinetics and it reduces the dose frequency. This feature is associated with improved patient compliance and thus improves the therapy.

d) Targeted therapy for local ailments in the upper GIT: The prolonged and sustained administration of the drug from FDDS to the stomach may be useful for local therapy in the stomach.

e) Reduced fluctuations of drug concentration: The fluctuations in plasma drug concentration are minimized, and concentration-dependent adverse effects that are associated with peak concentrations can be prevented. This feature is of special importance for drugs with a narrow therapeutic index. That makes it possible to obtain certain selectivity in the elicited pharmacological effect of drugs that activate different types of receptors at different concentrations.

f) Reduced counter-activity of the body: Slow release of the drug into the body minimizes the counter activity leading to higher drug efficiency. 
g) Extended time over critical (effective) concentration: The sustained mode of administration enables extension of the time.

h) Improved receptor activation selectivity: FDDS reduces the drug concentration fluctuation over a critical concentration and thus enhances the pharmacological effects and improves the clinical outcomes.

i) Minimized adverse activity at the colon: Retention of the drug in GRDF at stomach minimizes the amount of drugs that reaches the colon and hence prevents the degradation of drug that degraded in the colon

j) Site specific drug delivery: A floating dosage form is a widely accepted approach especially for drugs which have limited absorption sites in upper small intestine.

\section{Limitations/Disadvantages:}

a) These systems require a high level of fluid in the stomach for drug delivery tom float and work efficiently-coat, water.

b) Not suitable for drugs that have solubility or stability problem in GIT.

c) Drugs such as Nifedipine which is well absorbed along the entire GIT and which undergoes first pass metabolism, may not be desirable.

d) Drugs which are irritant to gastric mucosa are also not desirable or suitable.

e) The drug substances that are unstable in the acidic environment of the stomach are not suitable candidates to be incorporated in the systems.

f) The dosage form should be administered with a full glass of water $(200-250 \mathrm{ml})$. g) These systems do not offer significant advantages over the conventional dosage forms for drugs, which are absorbed throughout the gastrointestinal tract

Mechanism of Floating Systems: Floating drug delivery systems (FDDS) have a bulk density less than gastric fluids and so remain buoyant in the stomach without affecting the gastric emptying rate for a prolonged period of time. While the system is floating on the gastric contents (given in Figure 8 (a)), the drug is released slowly at the desired rate from the system.

After release of drug, the residual system is emptied from the stomach. This results in an increased GRT and a better control of the fluctuations in plasma drug concentration.

However, besides a minimal gastric content needed to allow the proper achievement of the buoyancy retention principle, a minimal level of floating force $(F)$ is also required to keep the dosage form reliably buoyant on the surface of the meal. To measure the floating force kinetics, a novel apparatus for determination of resultant weight has been reported in the literature.

The apparatus operates by measuring continuously the force equivalent to $\mathrm{F}$ (as a function of time) that is required to maintain the submerged object. The object floats better if $\mathrm{F}$ is on the higher positive side (Figure 8(b)).

This apparatus helps in optimizing FDDS with respect to stability and durability of floating forces produced in order to prevent the drawbacks of unforeseeable intra gastric buoyancy capability variations.

$\mathrm{F}=\mathrm{F}_{\text {buoyancy }}-\mathrm{F}_{\text {gravity }}$

$=(\mathrm{Df}-\mathrm{Ds}) \mathrm{gv}$

Where, $\mathrm{F}=$ total vertical force, $\mathrm{Df}=$ fluid density, Ds $=$ Object density, $\mathrm{v}=$ Volume andg $=$ acceleration due to gravity ${ }^{4}$ 


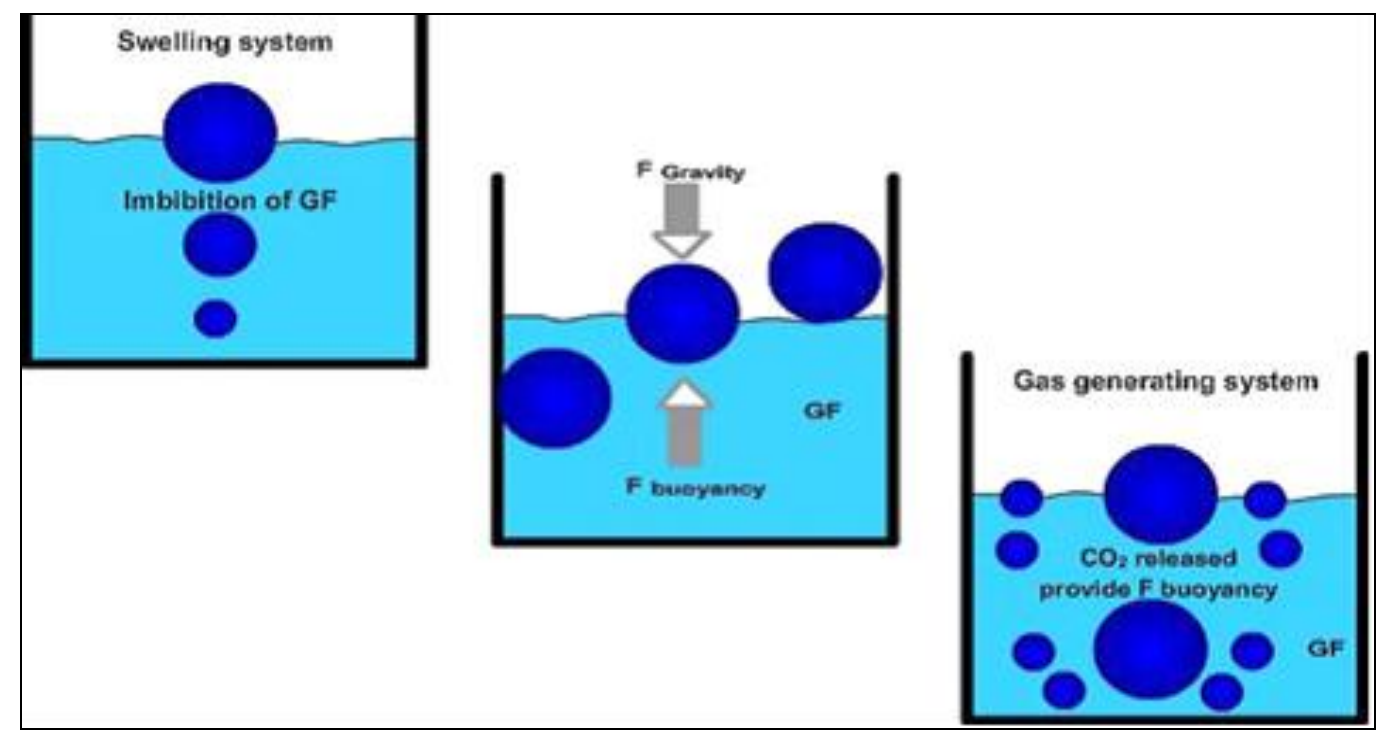

FIG. 8: MECHANISM OF FLOATING SYSTEM

\section{Drug Candidates Suitable for FDDS:}

a) Drugs that have narrow absorption window in GIT (e.g. L-DOPA, Para amino benzoic acid, furosemide, riboflavin). ${ }^{5}$

b) Drugs those are locally active in the stomach (e.g. misroprostol, antacids).

c) Drugs those are unstable in the intestinal or colonic environment (e.g. captopril, ranitidine $\mathrm{HCl}$, metronidazole) ${ }^{6}$.

d) Drugs that disturb normal colonic microbes (e.g. antibiotics used for the eradication of Helicobacter pylori, such as tetracycline, clarithromycin, amoxicillin).

e) Drugs that exhibit low solubility at high $\mathrm{pH}$ values (e.g. diazepam, chlordiazepoxide, verapamil) ${ }^{7}$.

\section{Factors Affecting Floating Drug Delivery System:}

1. Density: Density of the dosage form should be less than the gastric contents $(1.004 \mathrm{gm} / \mathrm{ml})$.

2. Size and Shape: Dosage form unit with a diameter of more than $7.5 \mathrm{~mm}$ are reported to have an increased GRT competed to with those with a diameter of $9.9 \mathrm{~mm}$. The dosage form with a shape tetrahedron and ring shape devises with a flexural modulus of 48 and 22.5 kilopond per square inch (KSI) are reported to have better GIT for 90 to $100 \%$ retention at 24 hours compared with other shapes. ${ }^{8}$

3. Fed or Unfed State: Under fasting conditions, the GI motility is characterized by periods of strong motor activity or the migrating myoelectric complexes (MMC) that occurs every 1.5 to 2 hours. The MMC sweeps undigested material from the stomach and if the timing of administration of the formulation coincides with that of the MMC, the GRT of the unit can be expected to be very short. However, in the fed state, MMC is delayed and GRT is considerably longer ${ }^{9}$.

4. Nature of the meal: Feeding of indigestible polymers of fatty acid salts can change the motility pattern of the stomach to a fed state, thus decreasing the gastric emptying rate and prolonging the drug release.

5. Caloric Content: GRT can be increased between 4 to 10 hours with a meal that is high in proteins ${ }^{10}$.

\section{Characterization Parameters:}

1. Size and Shape Evaluation: The particle size and shape plays a major role in determining solubility rate of the drugs and thus potentially its bioavailability. The particle size of the formulation is determined using Sieve analysis, Air elutriation analysis, Photo analysis, Optical 
microscope (Olympus (India) Pvt. Ltd), Electro résistance counting methods (Coulter counter), Sedimentation techniques, Laser diffraction methods, ultrasound attenuation spectroscopy, Air Pollution Emissions Measurements etc. ${ }^{11}$.

2. Floating Properties: Effect of formulation variables on the floating properties of gastric floating drug delivery system is determined by using continuous floating monitoring system and statistical experimental design ${ }^{12}$.

3. Surface Topography: The surface topography and structures are deter-mined using scanning electron microscope (SEM, JEOL JSM - 6701 F, Japan) operated with an acceleration voltage of 10k.v, Contact angle meter, Atomic force microscopy (AFM), Contact profiliometer.

4. Swelling Studies: Swelling studies were performed to calculate molecular parameters of swollen polymers. Swelling studies is determined by using Dissolution apparatus, optical microscopy and other sophisticated techniques which include H1NMR imaging, Confocal laser scanning micro and fats scopy (CLSM), Cryogenic scanning electron microscopy (Cryo-SEM), Light scattering imaging (LSI) etc. The swelling studies by using Dissolution apparatus (USP dissolution apparatus (usp-24) Lab India Disso 2000) was calculated as per the following formula ${ }^{13}$;

Swelling ratio $=$ Weight of wet formulation $/$ Weight of formulations

5. Determination of the Drug Content: Percentage drug content provides how much amount of the drug that was present in the formulation. It should not exceed the limits acquired by the standard monographs. Drug content is determined by using HPLC, HPTLC methods, Near infrared spectroscopy (NIRS), Micro titrimetric methods, Inductively Coupled Plasma Atomic Emission Spectrometer (ICPAES) and also by using spectroscopy techniques ${ }^{14}$.

6. Percentage Entrapment Efficiency: Percentage entrapment efficiency is reliable for quantifying the phase distribution of drug in the pre-pared formulations. Entrapment efficiency was deter-mined by using three methods such as Micro dialysis method, Ultra centrifugation, and pressure Ultra filtration.

7. In-vitro Release Studies: In vitro release studies (USP dissolution apparatus (usp-24) lab India disso 2000) are performed to provide the amount of the drug that is released at a definite time period. Release studies were performed by using Franz diffusion cell system and synthetic membrane as well as different types of dissolution apparatus ${ }^{15}$.

\section{Fourier Transforms Infrared Analysis:}

Fourier transform infrared spectroscopy (FTIR, Shimadzu, and Model-RT-IR-8300) is a technique mostly used to identify organic, polymeric, and some inorganic materials as well as for functional group determination. Fourier Transform Infrared Analysis (FT-IR) measurements of pure drug, polymer and drugloaded polymer formulations were obtained on FT-IR. The pellets were prepared on $\mathrm{KBr}$-press under hydraulic pressure of $150 \mathrm{~kg} / \mathrm{cm} 2$; the spectra were scanned over the wave number range of 3600 to $400 \mathrm{~cm}-1$ at the ambient temperature ${ }^{16}$.

\section{Differential Scanning Calorimetry (DSC):} DSC (Shimadzu, Model-DSC-60/DSC-50/ MetlerToldeo) are used to characterize water of hydration of pharmaceuticals. Thermo grams of formulated preparations are obtained using DSC instrument equipped with an intercooler. Indium/Zinc standards were used to calibrate the DSC temperature and enthalpy scale. The sample preparations were hermitically sealed in an aluminum pan and heated at a constant rate of $10^{\circ} \mathrm{C} / \mathrm{min}$; over a temperature range of $25^{\circ} \mathrm{C}$ $-65^{\circ} \mathrm{C}$. Inert atmosphere was maintained by purging nitrogen gas at the flow rate of $50 \mathrm{ml} / \mathrm{min}^{17}$.

\section{Application of Floating Drug Delivery Systems:} Floating drug delivery offers several applications for drugs having poor bioavailability because of the narrow absorption window in the upper part of the gastrointestinal tract. It retains the dosage form at the site of absorption and thus enhances the bioavailability. These are summarized as follows; 
a. Sustained Drug Delivery: HBS systems can remain in the stomach for long periods and hence can release the drug over a prolonged period of time. The problem of short gastric residence time encountered with an oral CR formulation hence can be overcome with these systems. These systems have a bulk density of $<1$ as a result of which they can float on the gastric contents. These systems are relatively large in size and passing from the pyloric opening is prohibited e.g.: Sustained release floating capsules of nicardipine hydrochloride were developed and were evaluated in vivo. The formulation compared with commercially available MICARD capsules using rabbits. Plasma concentration time curves showed a longer duration for administration (16 hours) in the sustained release floating capsules as compared with conventional MICARD capsules (8 hours) ${ }^{18}$.

b. Site-Specific Drug Delivery: These systems are particularly advantageous for drugs that are specifically absorbed from stomach or the proximal part of the small intestine (Riboflavin and Furosemide) e.g.: Furosemide is primarily absorbed from the stomach followed by the duodenum. It has been reported that a monolithic floating dosage form with prolonged gastric residence time was developed and the bioavailability was increased. AUC obtained with the floating tablets was approximately 1.8 times those of conventional furosemide tablets 19

c. Absorption Enhancement: Drugs that have poor bioavailability because of site specific absorption from the upper part of the gastrointestinal tract are potential candidates to be formulated as floating drug delivery systems, thereby maximizing their absorption e.g. a significantly increase in the bioavailability of floating dosage forms $(42.9 \%)$ could be achieved as compared with commercially available LASIX tablets (33.4\%) and enteric coated LASIX-long product $(29.5 \%)^{20}$.
ACKNOWLEDGEMENT: Author is thankful to Mr. Anurag Verma, Navneet Verma, Farogh Ahsan for their valuable guidance during preparing of this review article.

\section{REFERENCES:}

1. Tripathi GK, Singh S: Formulation and In vitro evaluation of $\mathrm{pH}$ sensitive oil entrapped polymeric blended buoyant beads of Amoxicillin. Scholar's research library 2010; 2(2): 131138.

2. Arora $\mathrm{S}$ et.al: Floating drug delivery system: A review. AAPS Pharm SciTech 2005; 6(3): 22-23.

3. Desai S, Bolton SA: Floating controlled release drug delivery system: in vitro- in vivo evaluation. Pharm Res 1993; 10:1321-1325.

4. Mathur P et al: Floating drug delivery system: An innovative acceptable approach in gastro retentive drug delivery. Scholars' research library 2010; 2(2):257-270

5. Garg R, Gupta GD: Progress in controlled gastro retentive delivery system. Trop J Pharm Res 2008; 7(3): 1055-1066.

6. Dave BS, Patel M: Gastro retentive drug delivery system of ranitidine HCL formulation and in-vitro evaluation. AAPS Pharm Sci Tech 2004; 235:87-54.

7. Shah SH,Patel JK, Patel NV: Stomach specific floating drug delivery system: A review. IntJ Pharm Tech Res 2009; 1(3): 623-633

8. Garima, Vishal and Arvind: Gastro retention: A means to address regional variability in intestinal drug absorption. Pharm Tech 2003; 27:50-68.

9. Talukder R,Fissihi R: Gastro retentive delivery system: A mini review. Drug DevInd Pharm Tech 2004; 30: 1019-1028.

10. Gopalakrishnan S, Chenthilnathan: A Floating drug delivery system: A review. J Pharm Sci Tech 2011; 3(2):548-554.

11. Vedhahari et al.: The recent developments on gastric floating drug delivery system: An overview. J PharmTech Res 2010; 2(1): 524-534.

12. Choi BY, Park HJHwang SJ: Preparation of alginate beads for floating drug delivery system effects of $\mathrm{CO}_{2}$ gas forming agents. Int J Pharm 2002; 239: 81-91.

13. Ferdous K, Selim R: Preparation and in-vitro evaluation of theophylline loaded gastro- retentive: Floating tablets of Methocel K4M. Dhaka Univ J PharmSci 2008; 7(1): 65-70.

14. Singh YT, Singh P., Rao: G Development and evaluation of floating microspheres of Verapamil hydrochloride. Brazilian J Pharm Sci 2007; 43 (4):529-534.

15. Vedha $\mathrm{H}$ et al.: The recent developments on gastric floating drug delivery system: an overview. Int $\mathrm{J}$ Pharm Tech Res 2010, 2(1): 524-534.

16. Arunachalam A et al.: Floating drug delivery system: A review. Int J Res PharmSci 2011; 2(1): 76-83.

17. Mayavanshi AV and Gajjar SS: Floating drug delivery system to increase gastric retention of drugs: A review. Res J Pharm Tech 2008; 1(4): 345-348.

18. Fell JT, Whitehead L and Collet H: Prolonged gastric retention using floating dosage forms. Pharm Tech 2000 24(3):82-90

19. Moursy NM, Afifi NH, Ghorab DM, El Saharty Y: Formulation and evaluation of sustained release floating capsules of Nicardipine hydrochloride. Pharm 2003; 58:38-43.

20. Garg S, Shringi S: Gastro retentive drug delivery systems Pharm Tech 2003: 160-166.

How to cite this article:

Tiwari V, Verma V and Verma N: Floating Drug Delivery System: A review. Int J Pharm Sci Res 2014; 5(7): 25962605.doi: 10.13040/IJPSR.0975-8232.5(7).2596-2605

This article can be downloaded to ANDROID OS based mobile. Scan QR Code using Code/Bar Scanner from your mobile. (Scanners are available on Google Playstore) 